Computing and Informatics, Vol. 38, 2019, 12101236 doi: 10.31577/cai_2019_5 1210

\title{
CASE STUDY ON HUMAN-ROBOT INTERACTION OF THE REMOTE-CONTROLLED SERVICE ROBOT FOR ELDERLY AND DISABLED CARE
}

\author{
Nayden Chivarov, Denis Chikurtev \\ Robotics Lab, European Polytechnical University \\ 23 Sv. Kiril i Metodiy Str., 2300 Pernik, Bulgaria \\ 83 \\ Institute of Information and Communication Technologies \\ Bulgarian Academy of Sciences \\ Acad. G. Bonchev St., Block 25A, 1113 Sofia, Bulgaria \\ e-mail: nshivarov@iinf.bas.bg, denis@iinf.bas.bg
}

\section{Stefan ChIvarov}

Institute of Mechanics and Mechatronics - IHRT, TU Vienna

Favoritenstrasse 9-11/E325 A4, A-1040 Vienna, Austria

e-mail: e11743488@student.tuwien.ac.at

\author{
Matus Pleva, Stanislav Ondas, Jozef Juhar \\ Department of Electronics and Multimedia Communications \\ FEI, Technical University of Košice \\ Park Komenského 13, 04120 Košice, Slovak Republic \\ e-mail: \{matus.pleva, stanislav.ondas, jozef.juhar\}@tuke.sk
}

\section{Kaloyan Yovchev}

Robotics Lab, European Polytechnical University 23 Sv. Kiril i Metodiy Str., 2300 Pernik, Bulgaria

Es

Faculty of Mathematics and Informatics, Sofia University

5 James Bourchier Blvd., 1164 Sofia, Bulgaria

e-mail: k.yovchev@fmi.uni-sofia.bg 
Abstract. The tendency of continuous aging of the population and the increasing number of people with mobility difficulties leads to increased research in the field of Assistive Service Robotics. These robots can help with daily life tasks such as reminding to take medications, serving food and drinks, controlling home appliances and even monitoring health status. When talking about assisting people in their homes, it should be noted that they will, most of the time, have to communicate with the robot themselves and be able to manage it so that they can get the most out of the robot's services. This research is focused on different methods of remote control of a mobile robot equipped with robotic manipulator. The research investigates in detail methods based on control via gestures, voice commands, and web-based graphical user interface. The capabilities of these methods for HumanRobot Interaction (HRI) have been explored in terms of usability. In this paper, we introduce a new version of the robot Robco 19, new leap motion sensor control of the robot and a new multi-channel control system. The paper presents methodology for performing the HRI experiments from human perception and summarizes the results in applications of the investigated remote control methods in real life scenarios.

Keywords: Service robots, human-robot interaction, remote control, speech recognition, gesture recognition, ROS

Mathematics Subject Classification 2010: 68-T40

\section{INTRODUCTION}

Prognoses of the United Nations show that there is a worldwide trend of continuously aging population and respectively increasing number of people with mobility difficulties [1]. Most of the elderly and disabled citizens want to live in their own houses [2] using the new smart home technologies for as long as possible, thus a robot will have to perform real-life interaction with them [3, 4]. The proposed remotecontrolled service robot for elderly and disabled care can help them with tasks of the every day life such as reminding them to take medications, serving food and drinks, turning on and off electronic devices, alerting when the user's health status is getting worse and connecting them with their physician, relatives or an emergency ambulance.

Assistive service robotics now expands as an alternative for improving the quality of life of elderly and disabled [5, 6]. From the data of the International Federation of Robotics it could be expected that in the period of 2019-2021 about 39 million of new service robots for personal use will be produced and about 34000 robots for the support of elderly and handicap assistance will be installed [7].

Remote control is widely used in many applications. The basic concept is that the robot should replace humans where people are exposed to unfavorable conditions or performing routine day-to-day activities [8, 9]. The essence of this robot 
management method is that the person controls the robot remotely, and the robot must possess the necessary qualities and functions to perform successfully in the intended tasks [10].

When talking about assisting people in their homes, it should be noted that they will, most of the time, have to communicate with the robot themselves and be able to manage it so that they can get the most out of the services the robot provides [11].

The aim of this study is to investigate the control methods for remote humanrobot interaction. The paper is structured as follows. Section 2 describes the hardware, software and control system of our robot "ROBCO 19". In Section 3, different methods for remote control of the robot are thoroughly investigated. The capabilities of these methods for human-robot interaction have been explored both in terms of utility and functionality. Section 4 presents the methodology for performing human perception experiments with the robot and the application of proposed remote control methods in real life scenarios. Section 5 describes the performed test scenarios and summarizes the results from the real life experiments.

\section{ASSISTIVE SERVICE ROBOT - ROBCO 19}

ROBCO 19 is the next iteration of the personal assistive robot "ROBCO 18" from 2018 [12. The robot was redesigned as follows. The laser scanner was replaced with a new RPLidar scanner which allows outdoor navigation. The sensor system was upgraded by adding an Intel RealSense camera for improved object recognition and manipulation. The microcontrollers have been upgraded with Teensy hardware. Also, a new graphical user interface and new software have been developed for autonomous navigation, collision avoidance and execution of predefined tasks. New electro-actuating systems (high power DC motor drivers), batteries and recharging docking system to allow $24 / 7$ service were installed. As a result, we have a remotecontrolled service robot for elderly and disabled care "ROBCO 19" (Figure 1).

The hardware components are described by their location in the layers of the mobile platform. In the first layer are DC motors, encoders, distance sensors, orientation sensor, batteries, controller and drivers. In the second layer are the computer of the robot and the laser scanner RPLidar. In the third layer are the articulated arm Mover4 and the RealSense camera.

Figure 2 shows the connections between robot devices. The robot's computer is connected to the mobile base controller, arm controller, RealSense and RPLidar over USB communication ports. The computer is the main computational device of the robot - sends/receives data, computes data, runs algorithms and controls the robot.

RPLidar is used for the autonomous navigation of the robot [13, 14]. The Lidar scans the area around the robot in 2D. The scanned parameters are described in Table 1. Thanks to the Lidar, the navigation system easily finds the robot's location and navigates smoothly and safely [15]. 


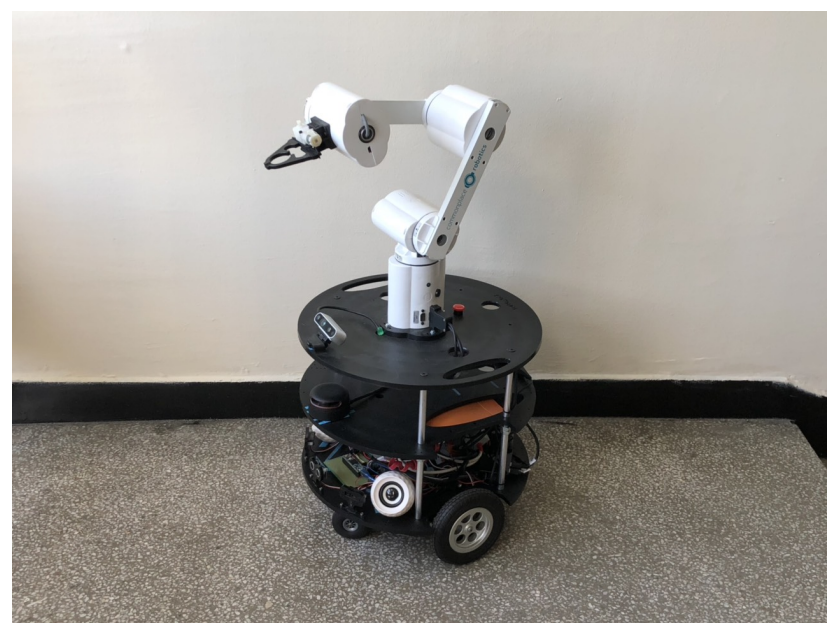

Figure 1. The service robot ROBCO 19

The RealSense camera is used for the robot's vision system. When we combine its depth sensor with its camera, we can recognize objects and locate their position in 3D space [16]. This property is very important for autonomous grasping of objects, for human recognition, and for autonomous navigation [17].

The Teensy controller works also on a separate level. It reads and converts the signals from the encoders, distance sensors and MPU (Motion Processing Unit),

\begin{tabular}{|c|c|}
\hline Device & Description/Characteristics \\
\hline Computer & Intel Xeon E3-1230 v5, 8 GB RAM \\
\hline Mobile base controller & $\begin{array}{l}\text { Teensy } 5.2 \text {, based on the MK20DX256 32-bit } \\
\text { ARMCortex-M } 4 \text { and } 72 \mathrm{MHz} \text { CPU }\end{array}$ \\
\hline Arm controller & PCAN-USB \\
\hline RealSense camera & $\begin{array}{l}\text { Depth Stream Resolution and FpS: } 1280 \times 720 \text {, } \\
90 \mathrm{fps} \text {, Depth Distance: Min: } 0.1 \mathrm{~m} \text {, Max: } 10 \mathrm{~m} \text {, } \\
\text { RGB Resolution and FpS: } 1920 \times 1080 \text { at } 30 \mathrm{fps} \text {. }\end{array}$ \\
\hline RPLidar scanner & $\begin{array}{l}\text { Distance Range: } 25 \text { meters, Sample Rate: } \\
16000 \mathrm{fps} \text {, Scan Rate: } 15 \mathrm{~Hz} \text { (adjustable be- } \\
\text { tween } 10-20 \mathrm{~Hz} \text { ), Angular Resolution: } 0.3375 \\
\text { degrees, Communication Speed: } 256 \mathrm{kbps} \text {. }\end{array}$ \\
\hline MPU - Motion Processing Unit & $\begin{array}{l}\text { MPU-9250 Nine-Axis (Gyro + Accelerometer + } \\
\text { Compass) MEMS Motion Tracking Device }\end{array}$ \\
\hline Drivers and motors & $12 \mathrm{~V}-10 \mathrm{~A}$ drivers, $12 \mathrm{~V}$ DC motors with gears \\
\hline Mover4 robotic arm & $\begin{array}{l}\text { Commonplace Robotics } \mathrm{GmbH} \text {, four degrees of } \\
\text { freedom, planar kinematic structure }\end{array}$ \\
\hline
\end{tabular}

Table 1. Specific characteristics of the robot's hardware components 
then sends the data to the PC. The controller receives data back from the robot's computer for running the platform, i.e. controlling the wheels. Then Teensy sends control signals to the drivers using Pulse Width Modulation (PWM).

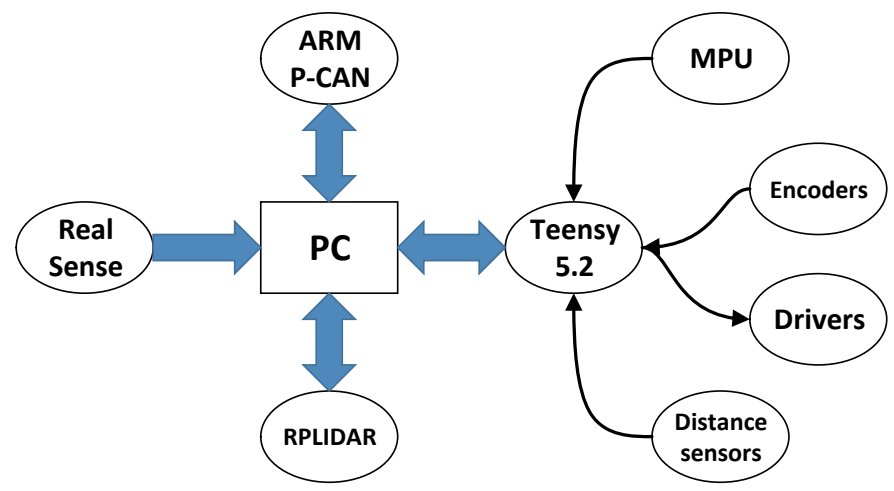

Figure 2. Hardware system of the robot

\subsection{Mobile Robot Platform}

A differential drive robot is a wheeled robot with two controllable wheels, as shown in Figure 3. To maneuver any differential drive robot on a plane, the robot needs a linear velocity $V$ and a heading $\theta$. By controlling the velocity and orientation, the path of the robot can be planned.

While we can vary the velocity of each wheel, for the robot to perform a side turn, the platform must rotate about a point that lies along the left and right wheels' common axis. The point that the robot rotates about is known as the ICC Instantaneous Center of Curvature.

By varying the velocities of the two wheels, we can vary the trajectories that the robot takes. Because the rate of rotation $\omega$ about the ICC must be the same for both wheels, we can write the following equations:

$$
\begin{aligned}
& \omega\left(R+\frac{l}{2}\right)=V_{r}, \\
& \omega\left(R-\frac{l}{2}\right)=V_{l}
\end{aligned}
$$

where $l$ is the distance between the centers of the two wheels, $V_{r}, V_{l}$ are the right and left wheel velocities along the ground, and $R$ is the signed distance from the ICC to the midpoint between the wheels. At any instance in time we can solve for $R$ and $\omega$ :

$$
R=\frac{l}{2} \frac{V_{l}+V_{r}}{V_{r}-V_{l}} ; \quad \omega=\frac{V_{r}-V_{l}}{l} .
$$




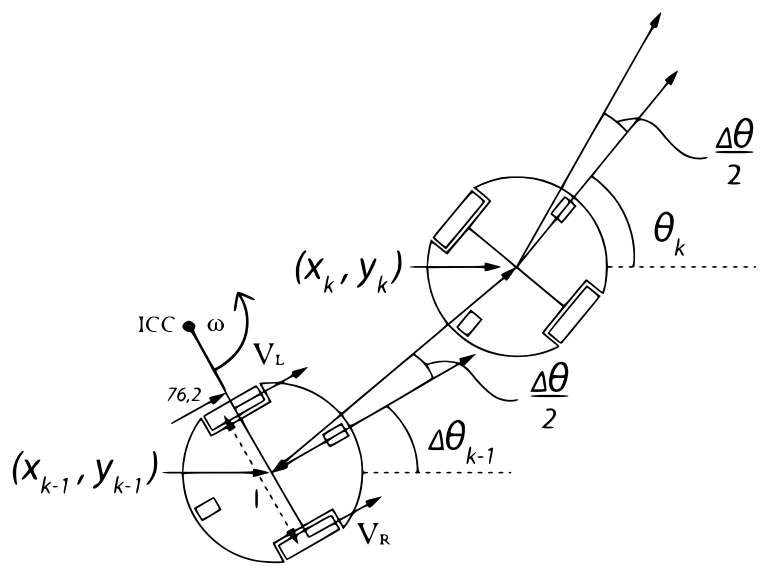

Figure 3. Differential drive mobile platform

There are three interesting cases with this kind of drive:

1. If $V_{l}=V_{r}$, then we have a linear forward motion in a straight line. $R$ becomes infinite, and there is effectively no rotation $-\omega$ is zero.

2. If $V_{l}=-V_{r}$, then $R=0$, and we have rotation about the midpoint of the wheel axis - we rotate in place.

3. If $V_{l}=0$, then we have rotation about the left wheel. In this case $R=\frac{l}{2}$. The same is true if $V_{r}=0$.

In Figure 3, assume the robot is at some position $(x ; y)$, headed in a direction forming an angle $\theta$ with the $X$ axis. We assume the robot's center is at a point in the middle of the wheel axle. By manipulating the control parameters $V_{l}$; $V_{r}$, we can get the robot to move to different positions and orientations. Please note that $V_{l}$ and $V_{r}$ are wheel velocities along the ground.

Knowing velocities $V_{l}$ and $V_{r}$ we can find the ICC's location using: $I C C=$ $[x-R \sin \theta, y-R \cos \theta]$ and at time $t+\delta t$ the robot's position will be:

$$
\left[\begin{array}{l}
x^{\prime} \\
y^{\prime} \\
\theta^{\prime}
\end{array}\right]=\left[\begin{array}{ccc}
\cos (\omega \delta t) & -\sin (\omega \delta t) & 0 \\
\sin (\omega \delta t) & \cos (\omega \delta t) & 0 \\
0 & 0 & 1
\end{array}\right]\left[\begin{array}{c}
x-I C C_{x} \\
y-I C C_{y} \\
\theta
\end{array}\right]+\left[\begin{array}{c}
I C C_{x} \\
I C C_{y} \\
\omega \delta t
\end{array}\right] .
$$

This equation simply describes the motion of the robot rotating at a distance $R$ about its ICC with an angular velocity of $\omega$.

\subsection{Articulated Robotic Arm}

The MOVER 4 robot of Commonplace Robotics GmbH has four degrees of freedom and planar kinematic structure [18]. The Table 2 lists the kinematic parameter 
values of the MOVER 4. Three of the degrees of mobility provide the positioning, and the fourth orientates the End Effector (EE) relative to the Z-axis of the coordinate system associated with the base of the robot. The coordinates of any point in the kinematic chain, and in particular the EE, can be derived from geometric considerations.

\begin{tabular}{|r|c|r|r|l|}
\hline Joint Number $\mathbf{n}$ & $\begin{array}{c}\text { Constraints } \\
\theta_{n}[\mathbf{D E G}]\end{array}$ & $\begin{array}{r}\text { Length } \\
L_{n}[\mathbf{m}]\end{array}$ & $\begin{array}{r}\text { Twist Angle } \\
\alpha_{n}[\mathbf{D E G}]\end{array}$ & $\begin{array}{l}\text { Offset } \\
S_{n}[\mathbf{m}]\end{array}$ \\
\hline \hline 1 & $-150,150$ & 0 & 90 & 0.206 \\
\hline 2 & $-50,65$ & 0.19 & 0 & 0 \\
\hline 3 & $-110,140$ & 0.22 & 0 & 0 \\
\hline 4 & $-140,135$ & 0.095 & 0 & 0 \\
\hline
\end{tabular}

Table 2. Kinematic parameters

If $q 1, q 2, q 3$ and $q 4$ are the generalized state space coordinates of the robotic manipulator, then the equations for the $(X, Y, Z)$-coordinates of the EE are as follows:

$$
\begin{aligned}
& X=\cos \left(q_{1}\right) \cdot\left(\cos \left(q_{2}\right) \cdot L_{2}+\cos \left(q_{2}+q_{3}\right) \cdot L_{3}+\cos \left(q_{2}+q_{3}+q_{4}\right) \cdot L_{4}\right), \\
& Y=\sin \left(q_{1}\right) \cdot\left(\cos \left(q_{2}\right) \cdot L_{2}+\cos \left(q_{2}+q_{3}\right) \cdot L_{3}+\cos \left(q_{2}+q_{3}+q_{4}\right) \cdot L_{4}\right), \\
& Z=S_{1}+\sin \left(q_{2}\right) \cdot L_{2}+\sin \left(q_{2}+q_{3}\right) \cdot L_{3}+\sin \left(q_{2}+q_{3}+q_{4}\right) \cdot L_{4} .
\end{aligned}
$$

\subsection{Control Software}

ROS is a meta-operation system for robot control [19]. ROS provides access to a number of open source packages that provide various applications and features 20 , 21]. This section describes the properties and features of some of the ROS packages that we use to control our service robot. The following packages are presented: SLAM, MoveIt, Robot node, ROS-bridge, Web_sockets, RPLidar, LeapMotion node, and Kinect node. Each package performs specific functions according to its purpose, and all nodes are connected to each other via the ROS Master.

Robot_node is the main node for controlling the robot. It calculates the specific parameters for each robot. All other nodes are connected to this node. For the robot described in this article, this package performs the following functions: reading the robot model, calculating speed (accelerometer), reading data from all sensors, reading incoming data from other nodes, and sending control data to the control engine (Teensy) of the mobile platform and the arm.

The code of this package describes the characteristics of the robot's mobile platform such as wheel diameter, platform width and length, encoder resolution, mobile platform type (two/four-wheel drive, differential/Mecanum/Omni drive), sensor location, drive controllers, and others. The diagram of connections between the nodes and their functions is in Figure 4 .

ROBCO 19 has three main operation modes: 


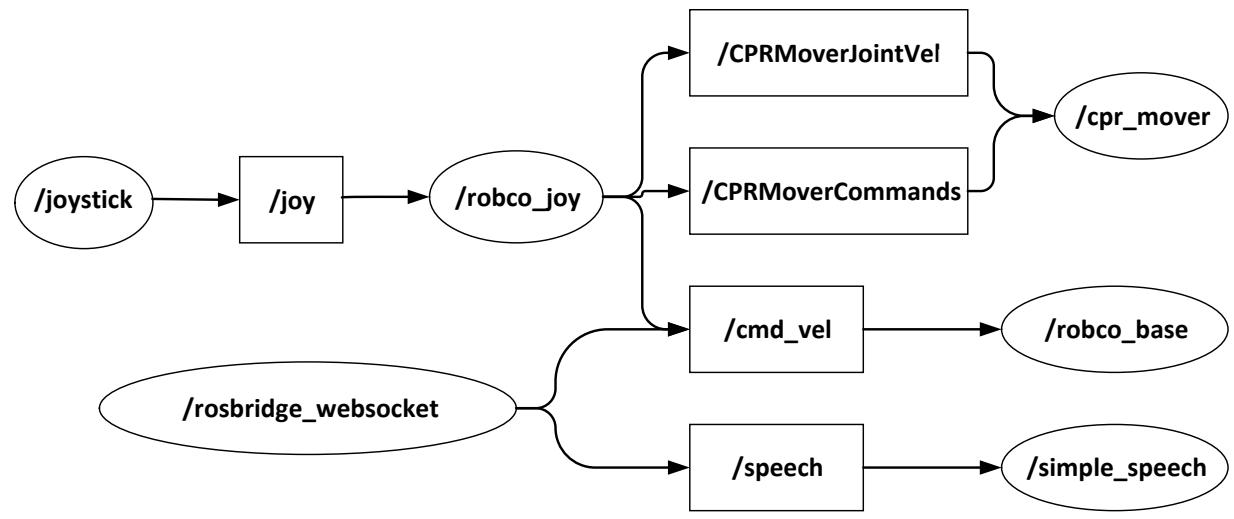

Figure 4. Node connection diagram

Manual Mode: In this mode, the robot receives commands from the user through the available control methods. All algorithms and programs for automated tasks are stopped. The user has full control of the robot motions but has to do all the command work to perform a task. ROBCO 19 has a user-friendly web-based User Interface for remote control [31, 32]. The robot can be controlled either by joystick, tablet/computer/phone (via WEB interface), voice or gestures.

Semi-Autonomous Mode: This mode combines Manual Mode with algorithms and programs for automated functions. Some of the tasks are predefined and the user only has to choose which task to be performed. FlexBe behavior engine's user interface is used for this purpose, which allows executing high-level tasks while the operator is able to influence the execution during runtime.

Autonomous Mode: The Autonomous mode requires only a single command from the user, then the robot performs all the necessary tasks until the goal is completed. It also uses FlexBe behavior engine which allows for fully autonomous execution of tasks and behaviors.

FlexBE is a powerful and user-friendly high-level behavior engine for generating complex robot behaviors without the need to manually code them. Among its basic capabilities, which interface standard functionality or your own system-specific features, state machines can be easily composed via the provided drag \& drop editor 33 .

To switch between operating modes we have added special buttons in the Web User Interface. This makes it very easy for the user or operator to change modes.

\section{METHODS FOR HUMAN-ROBOT INTERACTION}

Because we investigate human-robot interaction, various methods of controlling a service robot have been developed and explored. Therefore, the creation of a multi- 
channel (multi-modal) architecture has to be established to allow the robot to be controlled by all different methods at the same time [22]. In order to avoid conflicts at this stage, the structure itself gives each method a certain priority. The remote control channels - hardware and software joystick - have the highest priority. Following are the channels of control by gestures and voice. With the lowest priority of the direct-control channels is the Web-based control. Channel prioritization is described in detail in 12. This architecture is easy applicable and re-configurable when working with ROS because ROS works on the principle of Internet protocols. Thus, each method publishes commands through a different channel, but to the same subscriber. The multi-channel system monitors and manages data traffic.

\subsection{Gesture Control via Kinect Sensor}

In order to achieve convenience for different robot users, we have developed remote control methods through different devices. Gesture control is intended to replace standard voice commands because some elderly and disabled people have speech defects or are mute/deaf. Human-robot interaction through gestures is a good alternative and also helps maintain and develop motor skills of the users [23].

Control of the robot by recognition of hand gestures was implemented using the Kinect sensor.

Kinect is a sensor consisting of an RGB camera and a depth sensor. It provides functions to recognize the human skeleton and to monitor the positions of the joints of the whole body 1 . We have developed an algorithm and a program to process the data from human hands, and with processing we can control the robot's mobile platform or its articulated arm.

A particular feature of this type of control is that the user gives commands to the robot through a separate computer. The Kinect is connected to the user's computer and the user remotely controls the robot with hand movements.

The essence of this method is the following: Once the user is in front of the Kinect and all the joints of the hands and the head are recognized, the program starts working. As feedback for the user, the program displays specific words in the window menu: Working, Forward, Stop and others. The principle of operation, different zones that we have set empirically, and on which part the control is applied are shown in Figure 5 .

Actually, there are two modes of operation: sitting or standing in front of the Kinect sensor. The standing one was not preferred by the users, but it could be used for rehabilitation purposes.

To identify the different directions for the mobile platform, we have identified nine hand positioning zones. The inactive/passive zone is in the body area so that it covers the natural position of the hands when we are seated. When the hands fall

1 You can see all the skeleton points in Figure 2 of [24] article https://www. sciencepubco.com/index.php/ijet/article/download/10152/3614 


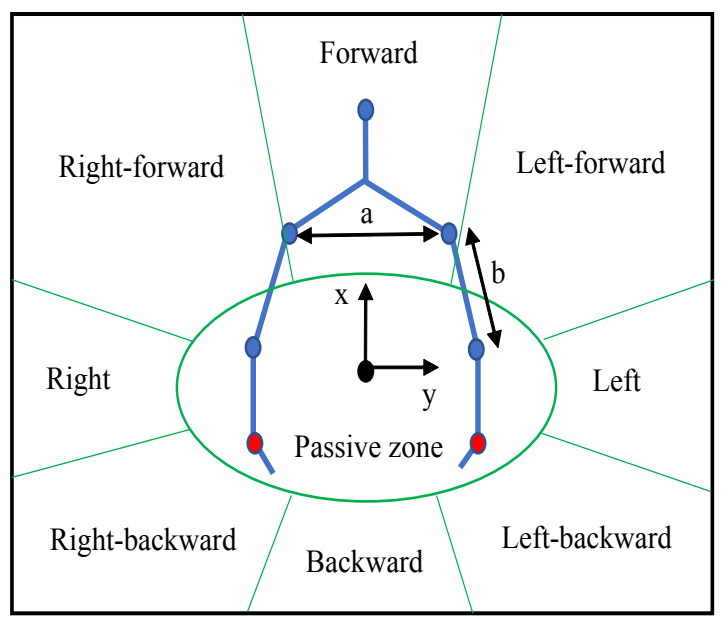

Figure 5. Control areas and dimensions of the measured parameters

into this zone, we send a stop command. When the user moves one of their hands to another zone, commands are then sent to the robot.

In the forward, backward, left and right zones, we send commands for linear motion or rotation in place, with control of the speed - as the wrist joint is closer to the inactive/passive zone, the lower is the speed. When the user's arm is in the zones like right-forward or left-backward, the control is based on the differential drive principle. The velocities change depending on the distance to the inactive/passive zone, the ratio of the linear/angular velocity depends on the ratio of the coordinates $x$ and $y$.

An important feature of this method is that the program should be configured according to the mobility of the user. Then, all zones are automatically determined according to the length between the joints. Initially, the inactive/passive zone is specified. It is defined as an ellipse with a radius of $x$ and $y$. Based on the parameters of the inactive/passive zone the parameters of the other zones are set. In this way, the problem of the different height of people is solved. For people with longer hands the zones have larger dimensions and vice versa.

The proportion is determined by taking half the distance between the shoulders $a$ and adding half of the relative length of the arms from the shoulder to the elbow $b$ to set the $y$ radius. For determining the radius by $x$, only half of $a$ is taken. The center of the global map is the center of the global coordinate system. So, the control parameters for the robot are: $v=f(x) * k ; \omega=f(y) * m$, where $k$ and $m$ are proportional coefficients.

Another important problem is the simultaneous submission of commands with both hands in opposite directions. In this case, a safety algorithm is implemented. If the user gives a forward command with both hands, the lower values are taken. If 
one hand is only forward and the other is forward and left/right, then the angular values are added. In case of opposite commands, both left and right or back and forth, we send a "stop" command.

\subsection{Gesture Control via Leap Motion Sensor}

Leap Motion is a stereo camera sensor and its main task is to recognize the human hand. Its main features are recognition of the position of the palm and each individual finger in 3D coordinates $[28,29,30$. In this way, we can recognize whether a fist is closed and how many fingers are folded or extended. All of these data can be used to control a mobile robot or articulated arm. We have the option to choose a method, criterion, or a complex set of several criteria for extracting data and converting them into control signals.

The prerequisite for running the program is to have a valid hand recognized by the sensor itself, otherwise no commands are given. To start sending commands, when the above condition is already met, the user has to close his hand into a fist first. These considerations have been made because of the need to ensure safety.

The method under development includes the following. Initially, we recognize if there is a hand over the sensor, then we take the palm coordinates and begin to convert them so that correct control signals are generated for the mobile platform and the robot's arm. Currently the control of the mobile platform and the arm is separated in two different programs. It is planned to integrate the two programs by introducing the option of switching the controlled device after a certain gesture.

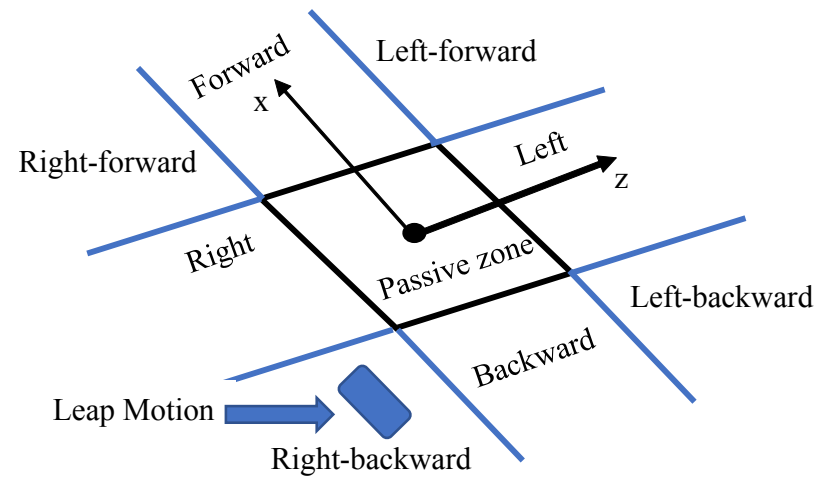

Figure 6. Working area in Cartesian coordinates for control of the mobile base using Leap Motion Sensor on the table

To control the mobile platform, linear and angular velocity have to be submitted. Since we get the values (from 0 to $300 \mathrm{~mm}$ ) of $x, y$ and $z$ coordinates from the palm position output data (Figure 6), it is relatively easy to convert these values into mobile platform control data using only the data obtained from $x$ and $z$. When the 
hand shifts out of the inactive/passive zone, we assign the linear velocity $v$ to $x$, and from the value of $z$ we assign the angular velocity $\omega$.

When handling the anthropomorphic manipulator, we use the $3 \mathrm{D}$ coordinates of the palm. The goal is to position the gripper of the manipulator by computing the necessary rotation of all its joints. To achieve this goal, we initially identify the passive zone where no commands are issued. Because this time we are working in 3 -dimensional space, this area is about the origin (zero) coordinate in the form of a cube with sides of $120 \mathrm{~mm}$ (Figure 7). As soon as the user's hand comes out of this zone, the transformed data $x, y, z$ of the palm are sent to the arm controller. In this case, we use the $x$ data to move forward and backward, $y$ to move up and down, and $z$ to move left and right. Accordingly, the combination of the three parameters gives us the final movement of the arm.

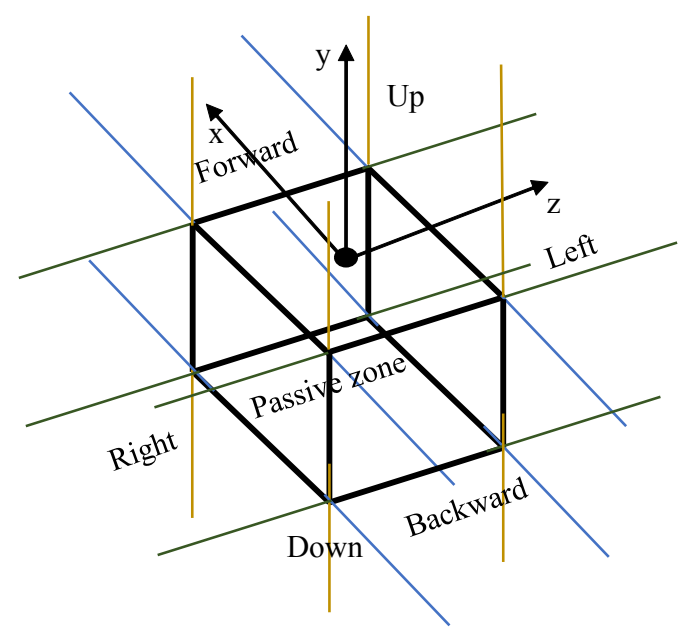

Figure 7. Working area in 3D for control of the Mover4

We multiply the values of $x, y, z$ by a factor of 0.5 in order to transform them into the actual speeds of the motors of the arm, because the range of the Mover 4 velocities is from 0 to $150 \mathrm{~mm} / \mathrm{s}$, and the range of the leap motion is measured from 0 to $300 \mathrm{~mm}$. The directions of rotation are determined by the equations for the inverse kinematics of the robot arm manipulator described in Section 2.2.

The system of equations for all parameters is:

$$
\begin{aligned}
& \omega_{0}=y * 0.5 ; \\
& \omega_{1}=(x *-0.5)+(z * 0.5) ; \\
& \omega_{2}=x *-0.5+(z * 0.5) ; \\
& \omega_{3}=x * 0.5+(z *-0.5) .
\end{aligned}
$$


To get closer to the natural human behavior, the grasping with the articulated arm is like palm grasping. When we close the palm, the gripper closes, and when we open the palm, the gripper opens. Again, for safety reasons when a valid hand is not detected or it is within the range of the passive zone, no commands are sent.

\subsection{Web User Interface}

A web-based user interface has been developed and added to obtain and manage robotic system status data (Figure 8). The main advantage of web-based interfaces is the ability to use any modern device that supports TCP/IP protocol and is on the same local network with the robot. Depending on network configuration there is also the possibility to control the system over the Internet. The web-based interface has visual click/touch design, allowing it to be used regardless of the type of the device - smartphone, tablet, laptop or full-sized desktop computer.

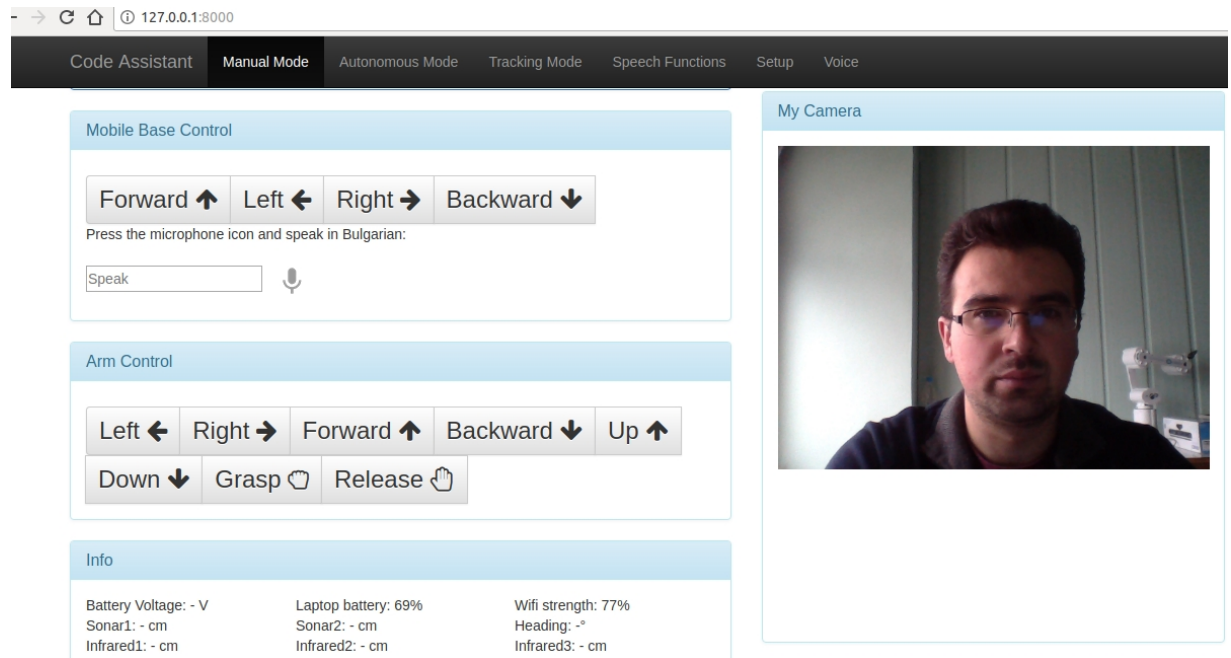

Figure 8. Part of the developed Web User Interface

The web-based interface is divided into separate sections depending on their functionality. The interface provides easy and convenient control of the robot in manual, semi-autonomous and autonomous mode. Commands and control signals can be sent via virtual buttons, a virtual joystick, or voice commands. The interface also provides the capability to monitor the robotic system as well as to configure robot's every parameter.

In the interface there are added buttons to control the movement of the mobile platform. Four basic moves are pre-programmed - forward/reverse linear movement, as well as left/right turn and rotational movement. Pressing these buttons once performs the corresponding movement at a preset and adjustable distance or angle. 
Buttons for manipulating the articulated robot arm manipulator are also provided. There are 8 of them together. Two of these buttons are to open and close the gripper. The other 6 buttons are for positioning the manipulator. The buttons perform upward, downward, left and right movement of the gripper, and also left and right rotation at the base of the manipulator. As with the mobile platform's buttons, the one-time press of these buttons performs motion with a preset and adjustable distance. The selected Mover4 robot manipulator has 4 degrees of freedom. This means that more than one actuator is used for the forward, backward, upward and downward movement of the gripper. For this reason, it is necessary to solve the kinematics of the manipulator and program it into the user interface.

A semi-autonomous map navigation feature has been added to the web-based user interface [34]. It is programmed as a separate section of the interface. It visualizes the map of the room created by the robot as well as the current position and orientation of the robot [35]. The UI allows the robot's current position and orientation to be kept in a specially created database at any time and named in a user-friendly way. From a convenient drop-down menu, the already saved database entries can be selected and submitted to the robot's control system. It will, then, navigate the robot all the way from the current to the assigned position.

The web-based user interface is a great feature for a robotic system which improves the overall user experience. It gives the user the comfort of controlling the robotic system directly by their favorite device instead of a separate specialized controller.

\subsection{Voice Control}

Speech to text conversion is used to trigger robot's different top level behaviors (FlexBe) or for manual control when in manual mode. Bulgarian speech recognition was implemented using the Google Cloud Speech-to-Text API [36]. ROBCO 19 must be connected to the Internet and the audio capture is transferred to Google servers for STT (Speech To Text) processing. The TTS (Text To Speech) synthesis in various languages is provided by the Espeak, open source software speech synthesizer. As there is no good quality synthesized Bulgarian language voice in Linux, we use VMware Virtual Windows machine to provide Bulgarian TTS, using the Windows SAPI (Speech Application Programming Interface).

For testing and simplicity we have developed predefined phrases for voice control. For example, "robot forward" means for the robot to move $0.3 \mathrm{~m}$ forward. Speech to text recognition is done by Google API and parsed using "word spotting". When the API returns the recognized text, we search it for the specific phrase using simple code in JavaScript. But the problem is that if you say for example "the robot goes forward" it also detects that there are the words "robot" and "forward" and it sends the commands for the movement. All these predefined phrases are for demonstration purpose, only to show that the robot can be controlled by voice commands and the Natural Language Understanding module (currently only "word spotting") will be improved for next releases. 
We can add more complicated actions for example, the phrase "robot go to the kitchen" should instruct the robot to go to a specific place at home. If we saved that location in the autonomous navigation then this phrase could be directly connected to the action of performing movement to the position in the kitchen. In addition, specific voice commands may activate one or more actions from the FlexBE engine.

For the issue of security and privacy it is required for some applications not to use the third party cloud services, which could store all the voice requests for future improvement of the service. For this purpose, we tested the Julius LVCSR (Large Vocabulary Continuous Speech Recognition) engine with freely available English acoustic models ${ }^{2}$ and fixed grammar [37]. However, for the proposed purpose of free phrase processing we will need more complex language models. Satisfactory models could be built for this purpose using freely available corpora of different languages using modern deep learning techniques for neural networks. More robust language models can be trained using a unique approach proposed in [38], where the methodology for training language models (LM) without training data is described.

The methodology relies on the iterative process, in which, at the beginning, an initial model is trained only from system vocabulary and randomly generated phrases, which summarize all devices, actions and functions of the robot. Then, the LM can be retrained using recognized phrases automatically or semi-automatically (after corrections made by a human expert). The final model then enables significantly higher number of phrases than fixed grammar. Also the detection of emotions from the recognized speech could be applied [39] for more natural human-like communication behavior.

We are currently working on a joint Slovak-Taiwan bilateral DeepSpeech project called Deep Learning for Advanced Speech Enabled Applications. Experience from using Kaldi, Tensorflow and DeepSpeech (Mozilla project with coincidentally the same acronym as our project) gives us the opportunity to build a high quality local Neural Network based speech recognition engine with higher accuracy than Julius based on finite-state transducers. For the LVCSR task, the Kaldi DNN approach achieved $8 \%$ Word Error Rate (percentage of incorrectly recognized words out of all words in a test set) in English and $17 \%$ in Slovak.

After combining Slovak and English using Language Identification Module the system was able to recognize English words with similar $8 \%$ WER and Slovak words with $16 \%$ WER. A similar approach could be used for English and Bulgarian language after gaining enough Bulgarian speech data and corpuses. Currently our Taiwanese partner from TaipeiTech is proposing a joint project with the European Polytechnical University (EPU) to work towards this goal [40].

After successful recognition of a continuous speech phrase, the module of Natural Language Understanding (NLU) needs to be in place to extract the intended meaning and Natural Language Generation (NLG) to prepare a meaningful sentence with the required response, if needed, for this application. Rule-based NLU module can be adopted from our previous solution described in [41].

2 https://sourceforge.net/projects/juliusmodels/ 
Usage of more complex voice commands can result in occurrence of ambiguities, which can be solved in dialogue interaction. To manage dialogue in task-oriented scenarios, the VoiceXML-based dialogue manager can be integrated. We designed and developed an advanced VoiceON unit, that implements interpretation of enhanced VoiceXML scripts (see 42]). Our modification of VoiceXML enables integrating robot action control directly through VoiceXML < prompt $>$ element. Moreover, VoiceXML integrates frame-based approach to natural language generation, which well fits the proposed scenario.

VoiceON dialogue manager supports JavaScript language and easy extension through calling own executables. Position of the robot can be obtained by calling a special object and then it can be inserted into the VoiceXML variables for further use. Variables can be stored in the application scope of the VoiceXML application and easily used to provide the position description in any state of the interaction.

\section{METHODOLOGY FOR USABILITY EXPERIMENTS}

The tests with elderly and disabled were performed during the project "Tele-controlled Service Robots for Increasing the Quality of Life of Elderly and Disabled, No. DN 07/23 - 15.12.2016" financed by the Bulgarian National Science Fund for the European Polytechnical University - Pernik, Bulgaria.

The tests have been conducted with 30 participants in two target groups, 15 elderly men and women and 15 disabled people using multi-channel robot management software for controlling ROBCO 19. They controlled the robot through virtual joystick, voice commands, mimic gestures and head movements. In the first project year the tests were performed with elderly people at the Scientist house of the Bulgarian Academy of Sciences (Figure 9). The elderly people involved in the experiments were between 66 and 81 years old (average 75.3 years old). For the second project year tests were performed with disabled volunteers at Union of the Disabled in Bulgaria, Trojan Branch (Figure 10). The disabled people were between 34 and 80 years old (average 63.8 years old) and with incapacity rate between $59 \%$ and $90 \%$ (average $78.2 \%$ ). The incapacity rate is determined by the Bulgarian National Expert Medical Commission.

There have been real tests of the proposed remote control of the mobile service robot performed with elderly and disabled people, including:

- Efficient support for the elderly and disabled at their different needs;

- Reminder when to take their medication;

- Food and beverage service;

- Switching on/off electronic devices;

- Alerting the doctor, relatives, or ambulance service with possible indicators of deterioration in health (day and night monitoring of elderly and disabled, described in details in [43]); 
1226 N. Chivarov, D. Chikurtev, S. Chivarov, M. Pleva, S. Ondas, J. Juhar, K. Yovchev

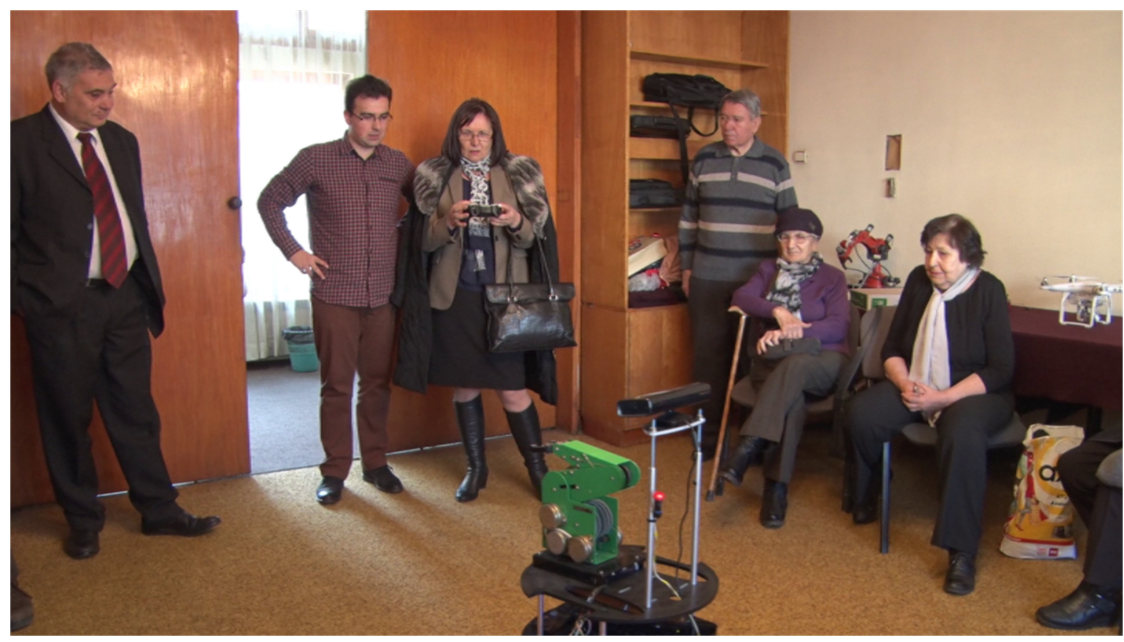

Figure 9. Performed tests with elderly

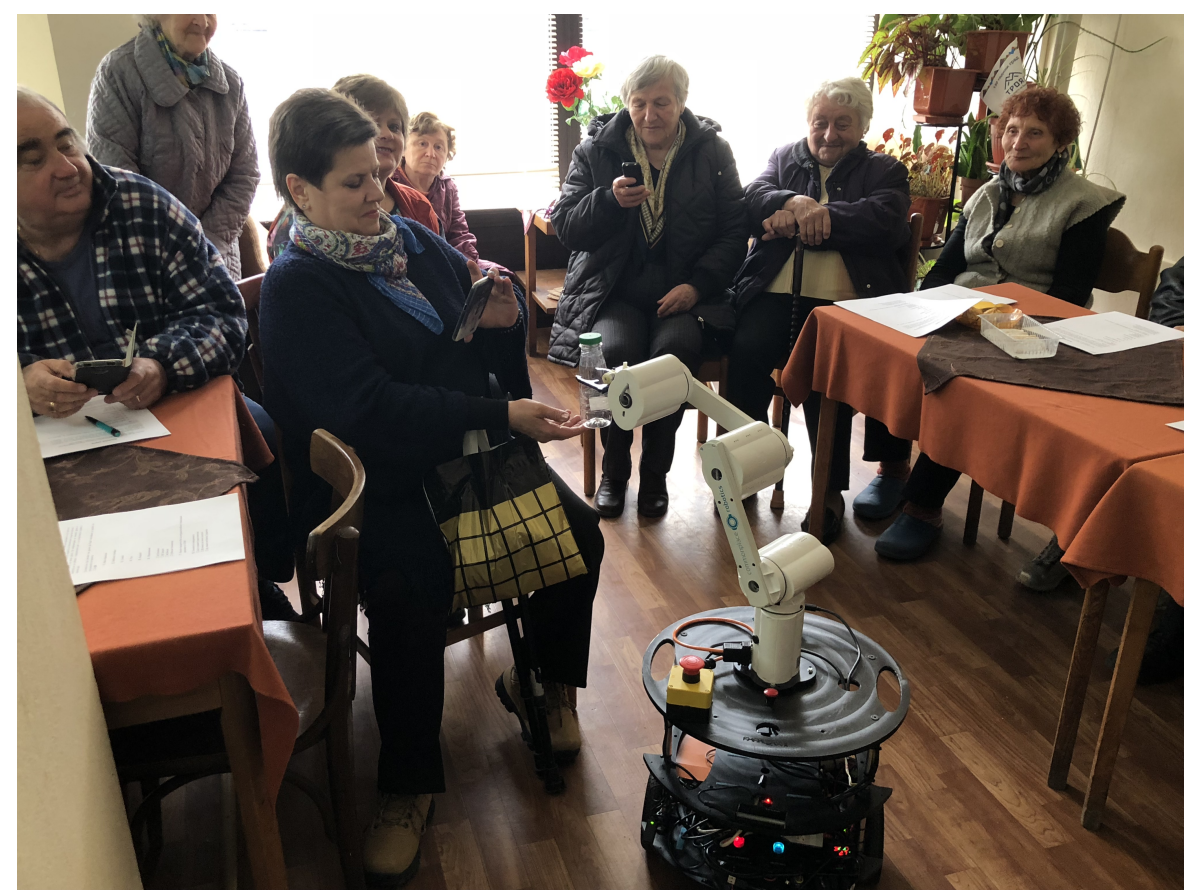

Figure 10. Performed tests with disabled 
It is important to note that no special education or technical knowledge is required to work with the robot. The purpose of these tests was to verify the application effectiveness and the reliability of the robot systems. In the experiment room, two tables were placed at a distance of about 3 to $4 \mathrm{~m}$ apart. On the first table there was: the computer and the smart-phone of the experiment lecturer, the manual control joystick of ROBCO 19, the Leap Motion and the Kinect. The tests duration with each of the target groups was one day.

In the frame of four academic hours in the morning, the lecturer has presented, demonstrated and trained all methods and modes for control of the robot to each target group. After the explanation and training, the lecturer prepared the robot for the experiments. On the opposite table there were household items for the robot to pick up such as: a $330 \mathrm{ml}$ plastic bottle of mineral water, a pack of biscuits, a medicine box, a small soft drink bottle and more objects weighing up to $250 \mathrm{~g}$. The goal for the elderly/disabled participants was to grasp one of the items on the table, take it to the other table and deliver it to someone of the other participants in the tests, thus the three operation modes were tested and evaluated by the users. All experiments were conducted under the same conditions, with some features described for each individual experiment. The facilitator had the task of turning on the robot and all necessary devices, as well as ensuring proper operation and safety.

Within four hours at the afternoon, the target groups members have used all of the presented control methods and operational modes of the robot, fulfilling the described above goal of the test. Each participant has chosen the appropriate methods and modes of robot control, depending of its personal preferences and motion difficulties. Finally, the groups of the elderly and disabled completed a questionnaire about their impressions and attitudes toward the robot. In the questionnaire more than one answer per question was allowed.

The study was conducted according to the project methodology and approved by the Ethics Committee, in coordination with Physiological Department of the European Polytechnical University (EPU). Ethics Committee was composed of the project leader and scientific members. The Committee in coordination with EPU Physiological Department has approved the methodology for the tests and the included questionnaires.

Under Regulation (EC) 2016/679 of the European Parliament and of the Council of 27 April 2016 on the protection of individuals with regard to the processing of personal data, test subjects were informed that the personal data in the survey were not collected for commercial purposes but would be used for statistical and scientific purposes.

The questionnaire results show that both elderly and disabled people expressed a positive feedback about using remote-controlled service robot ROBCO 19 for their needs, performing real interaction with the robot. Each participant conducted the tasks, using the chosen methods before the experiments.

In terms of functionality, they expect the robot to be able to monitor their health, bring heavy or hard-to-reach items, carry food, water and drugs, and contact the 


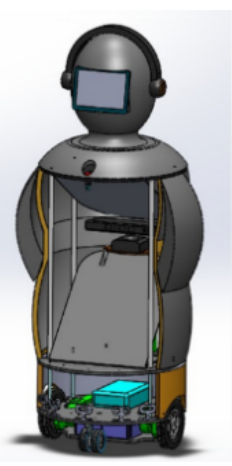

a)
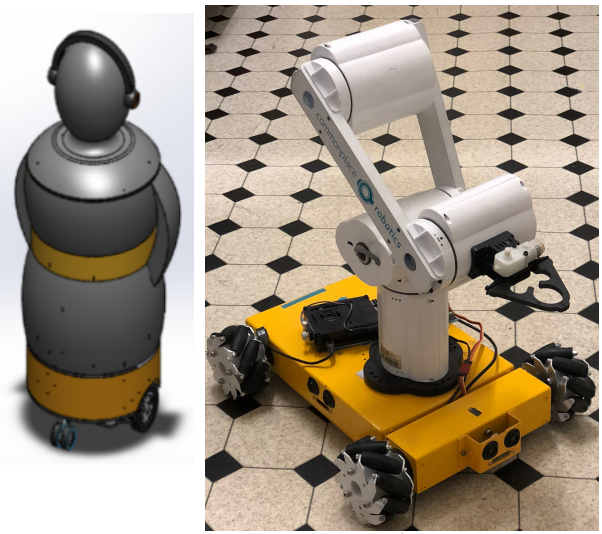

b)

Figure 11. a) Anthropomorphic (humanlike) design; b) Robco 20 - industrial design (in development with Mecanum wheels)

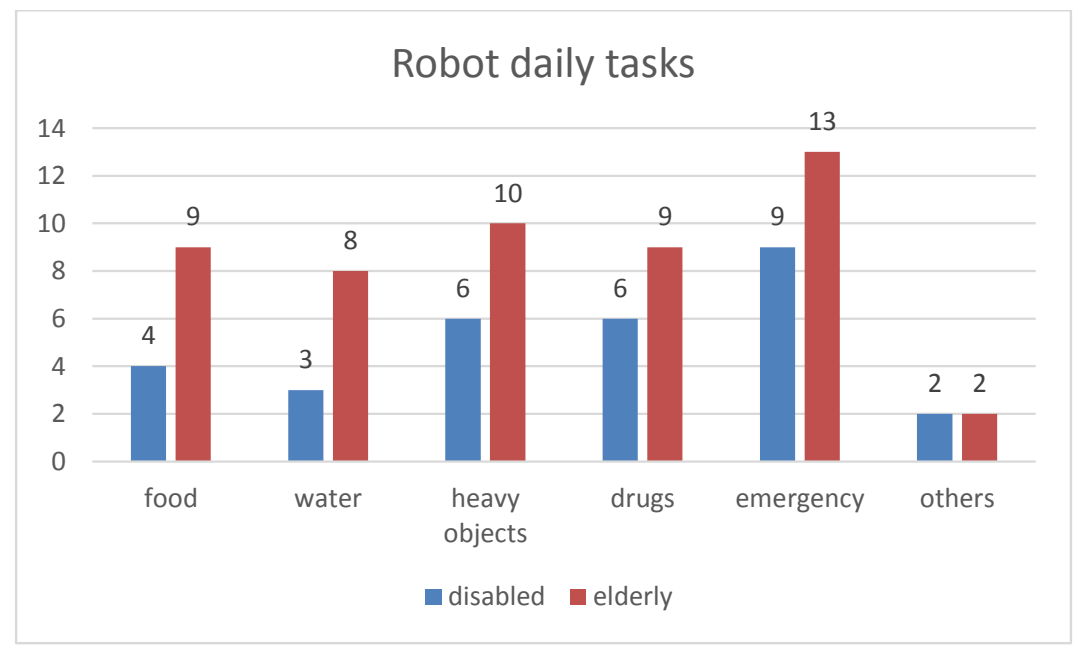

Figure 12. Robot daily tasks desired by the elderly and disabled

first aid in case of emergency (Figure 12). In the "others" section, volunteers have requested rehabilitation as an additional feature of the robot.

When asked about the industrial design, elderly participants (most of them living alone) preferred a human-like design (53\%) for the robot (Figure 11 a)d, while the disabled prefer more functional one (60\% for Robco 20) (Figure $11 \mathrm{~b}$ ). For the control of the robot, both elderly and disabled individuals prefer voice control (Figure 13). The most effective and less time consuming control methods are physical joystick (for the elderly) and virtual joystick embedded in the Web UI (for the 


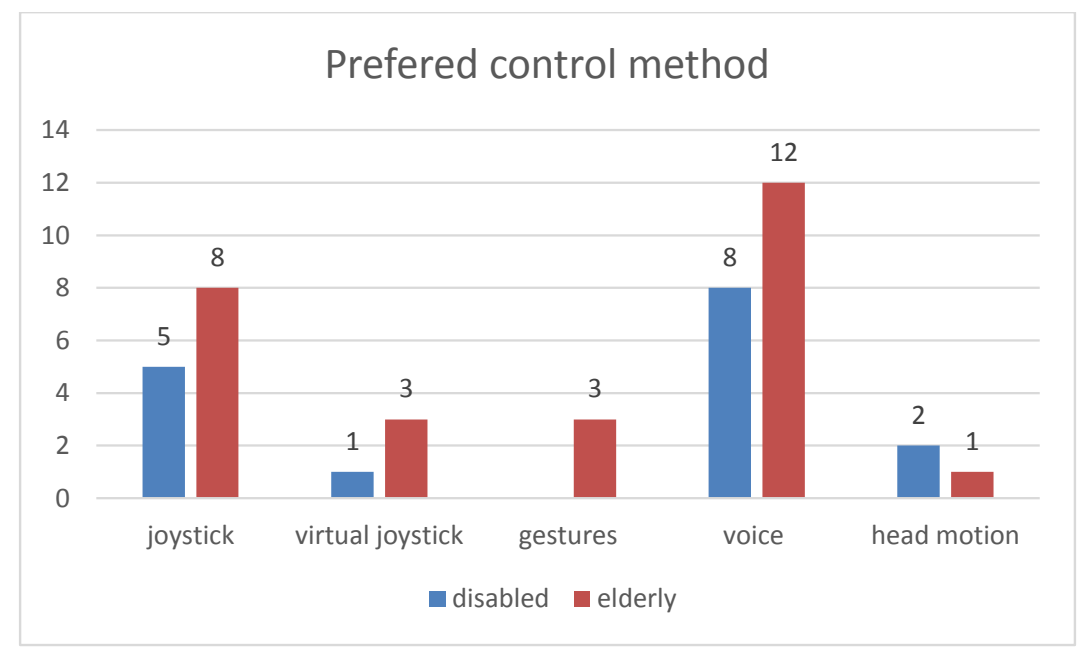

Figure 13. Preferred control method

disabled). For the voice control of the robot, they prefer the female voice (Figure 14.

Finally, elderly prefer to remote control the robot themselves, while disabled people would allow the robot to be controlled by their relatives, social caregivers and doctors as well (Figure 15). The most preferred operation mode is the manual mode, where users feels more secure and independent, while controlling the robot.

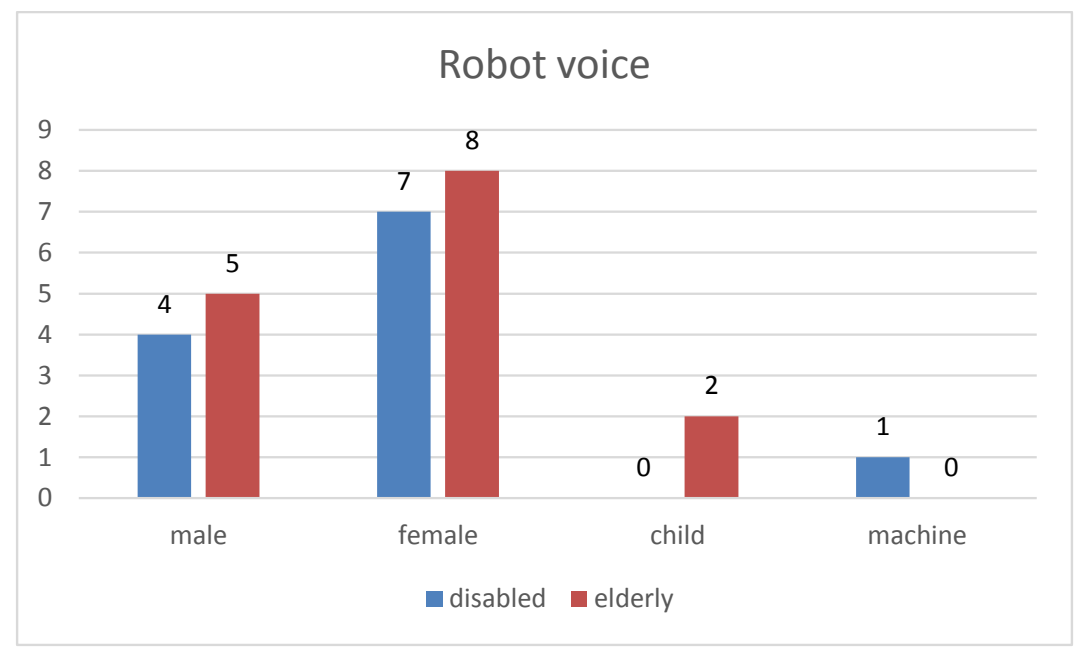

Figure 14. Preferred voice of the robot 


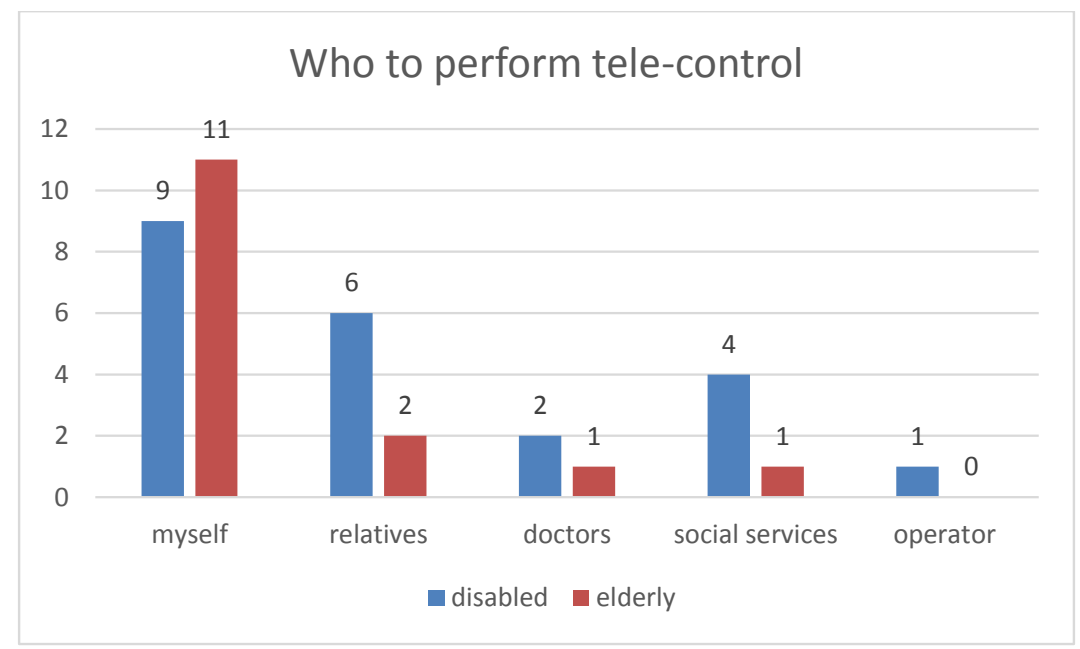

Figure 15. Preferred operator of the robot

\section{CONCLUSION}

This research involved a detailed investigation of different methods of remote humanrobot interaction: gesture control via Kinect and Leap Motion sensor, web-based user interface and voice-based control. These methods were applied to a mobile platform robot equipped with an articulated arm.

We have obtained useful information about what elderly and disabled people consider useful for their care from our robot. Interestingly, the elderly prefer to control the robot by themselves $(73 \%)$, but disabled people would like to do the remote control by somebody else $(57 \%)$ for instance their relatives or nurses. The results show that the described methods for remote control of service robots are convenient and easy to use. Experienced participants completed all tasks using different methods. The trial period was relatively short, indicating that the methods were natural and enjoyable for such application.

The two-thirds of participants prefer to control the robot by choosing the most appropriate method and mode for them. We can conclude that both elderly and disabled prefer a female voice and the joystick control methods. Due to their motion difficulties, elderly and disabled do not prefer gesture and head motion controls. The various control methods provide the opportunity for each user to choose the method which is the most convenient for him/her. The multi-channel control system allows the user to switch different control methods; thus, the user can select the preferred control method. The users did not prefer the standing mode of gesture control using the Kinect sensor because of fatigue. However, the mode still could be used for rehabilitation purposes and occasionally forced by the doctor. 
The new version of the robot - Robco 19, described in this paper, was successfully tested with real users and the new modern gesture control using the leap motion sensor, and finally, the proposed multi-channel (multi-modal) architecture was successfully implemented and evaluated.

For future work, we plan to research the following tasks: introducing a new function for bringing heavy and difficult to reach objects for manipulation, a functionality which would provide rehabilitation training, and other additional functions according to the results from the questionnaire. We believe that these additional features could greatly improve the robot system, which will become more useful providing new functionalities to make everyday life of the elderly and disabled citizens much easier.

\section{Acknowledgements}

The research was partially supported by the Bulgarian National Science Fund (grants No. DN 07/23-15.12.2016 and KP-06-M27/1-04.12.2018), by the Slovak Research and Development Agency (grants No. APVV SK-TW-2017-0005, APVV-15-0731 and APVV-15-0517) and by the Cultural and Educational Grant Agency financed by the Ministry of Education, Science, Research and Sport of the Slovak Republic (grant No. KEGA 009TUKE-4/2019).

\section{REFERENCES}

[1] Broekens, J.-Heerink, M.-Rosendal, H.: Assistive Social Robots in Elderly Care: A Review. Gerontechnology, Vol. 8, 2009, No. 2, pp. 94-103.

[2] Körtner, T.: Ethical Challenges in the Use of Social Service Robots for Elderly People. Zeitschrift für Gerontologie und Geriatrie, Vol. 49, 2016, No. 4, pp. 303-307, doi: 10.1007/s00391-016-1066-5

[3] Hosseini, S.-Goher, K.: Personal Care Robots for Older Adults: An Overview. Asian Social Science, Vol. 13, 2017, No. 1, pp. 11-19, doi: 10.5539/ass.v13n1p11.

[4] Portugal, D.-Santos, L.-Alvito, P.-Dias, J.-Samaras, G.Christodoulou, E.: SocialRobot: An Interactive Mobile Robot for Elderly Home Care. 2015 IEEE/SICE International Symposium on System Integration (SII 2015), Nagoya, IEEE, 2015, pp. 811-816, doi: 10.1109/SII.2015.7405084.

[5] Kachouie, R.-Sedighadeli, S.-Khosla, R.-Chu, M.-T.: Socially Assistive Robots in Elderly Care: A Mixed-Method Systematic Literature Review. International Journal of Human-Computer Interaction, Vol. 30, 2014, No. 5, pp. 369-393, doi: 10.1080/10447318.2013.873278

[6] Zielinska, T.: History of Service Robots. In: Ceccarelli, M. (Ed.): Service Robots and Robotics: Design and Application. IGI Global, 2012, pp. 1-14, doi: 10.4018/9781-4666-0291-5.ch001

[7] IFR, International Federation of Robotics, 2019. Retrieved May 21 ${ }^{\text {st }}$, 2019, from http://www.ifr.org/ 
[8] Chivarov, N.-Chikurtev, D.-Rangelov, I.-Markov, E.-Gigov, A.Shivarov, N.-Yovchev, K.-Miteva, L.: Usability Study of Tele-Controlled Service Robot for Increasing the Quality of Life of Elderly and Disabled - "ROBCO 17". In: Aspragathos, N., Koustoumpardis, P., Moulianitis, V. (Eds.): Advances in Service and Industrial Robotics (RAAD 2018). Springer, Cham, Mechanisms and Machine Science, Vol. 67, 2019, pp. 121-131, doi: 10.1007/978-3-030-00232-9_13.

[9] Kim, S.-C.-LeE, B.-K.-KIM, C.-Y.: Usability Evaluation of Communication Service Robot for the Elderly. Journal of Back and Musculoskeletal Rehabilitation, Vol. 32, 2019, No. 2, pp. 313-319, doi: 10.3233/BMR-169655.

[10] Zahariev, R.-Valchkova, N.-Angelov, G.-Paunski, Y.: Cognitive Service Mobile Robots for Help of Disabled People. Proceedings of the International Conference "Robotics and Mechatronics and Social Implementations" 2018. Complex Control Systems, Vol. 1, 2018, pp. 74-79.

[11] Mast, M.-Burmester, M.-Graf, B.-Weisshardt, F.-Arbeiter, G.Španěl, M.-Materna, Z.-Smrž, P.-Kronreif, G.: Design of the HumanRobot Interaction for a Semi-Autonomous Service Robot to Assist Elderly People. In: Wichert, R., Klausing, H. (Eds.): Ambient Assisted Living. Springer, Cham, Advanced Technologies and Societal Change, 2015, pp. 15-29, doi: 10.1007/978-3319-11866-6_2

[12] Chivarov, N.-Chikurtev, D.-Markov, E.-Chivarov, S.-Kopacek, P.: Cost Oriented Tele-Controlled Service Robot for Increasing the Quality of Life of Elderly and Disabled - ROBCO 18. IFAC-PapersOnLine, Vol. 51, 2018, No. 30, pp. 192-197, doi: 10.1016/j.ifacol.2018.11.285.

[13] Cheng, Y.-Wang, G. Y.: Mobile Robot Navigation Based on Lidar. 2018 Chinese Control and Decision Conference (CCDC), Shenyang, IEEE, 2018, pp. 1243-1246, doi: 10.1109/CCDC.2018.8407319.

[14] Ochndo, M. G.-Certad, N.-Alvarado, S.-Terrones, Á.: Autonomous 2D SLAM and 3D Mapping of an Environment Using a Single 2D LIDAR and ROS. 2017 Latin American Robotics Symposium (LARS) and 2017 Brazilian Symposium on Robotics (SBR), Curitiba, IEEE, 2017, pp. 1-6, doi: 10.1109/SBR-LARSR.2017.8215333.

[15] Chikurtev, D.: Indoor Navigation for Service Mobile Robots Using Robot Operating System (ROS). Problems of Engineering Cybernetics and Robotics, Vol. 67, Sofia, 2016, pp. 61-70.

[16] Babič, M.-Hluchy, L.-Krammer, P.-Matovič, B.-Kumar, R.Kovač, P.: New Method for Constructing a Visibility Graph-Network in 3D Space and a New Hybrid System of Modeling. Computing and Informatics, Vol. 36, 2017, No. 5, pp. 1107-1126, doi: 10.4149/cai_2017_5_1107

[17] Gatesichapakorn, S.-Takamatsu, J.-Ruchanurucks, M.: ROS Based Autonomous Mobile Robot Navigation Using 2D LiDAR and RGB-D Camera. 2019 First International Symposium on Instrumentation, Control, Artificial Intelligence, and Robotics (ICA-SYMP), Bangkok, Thailand, IEEE, 2019, pp. 151-154, doi: 10.1109/ICA-SYMP.2019.8645984

[18] Yankov, K.: Inverse Kinematics for Educational Robot MOVER 4. Applied Researches in Technics, Technologies and Education (ARTTE) - Journal of the Faculty 
of Technics and Technologies, Trakia University, Vol. 5, 2017, No. 3, pp. 212-224, doi: 10.15547/artte.2017.03.009.

[19] KoubÂA, A. (Ed.).: Robot Operating System (ROS). The Complete Reference (Volume 1). Springer, Cham, Studies in Computational Intelligence, Vol. 625, 2016, doi: 10.1007/978-3-319-26054-9.

[20] RaO, H. S.-Desai, V.H.-Bhat, R.-Jayaprakash, S.-Sampangi, Y.: A Study and Implementation of Mapping and Speech Recognition Techniques for an Autonomous Mobile Robot Based on ROS. International Journal of Advanced Mechatronic Systems, Vol. 7, 2017, No. 5, pp. 303-310, doi: 10.1504/IJAMECHS.2017.095874

[21] Hendrich, N.-Bistry, H.-Zhang, J.: Architecture and Software Design for a Service Robot in an Elderly-Care Scenario. Engineering, Vol. 1, 2015, No. 1, pp. 27-35, doi: 10.15302/J-ENG-2015007.

[22] Chivarov, S.-Chikurtev, D.-Yovchev, K.-Chivarov, N.: Multi-Channel Software Infrastructure for Remote Control of Service Robots. $20196^{\text {th }}$ International Conference on Control, Decision and Information Technologies (CoDIT), Paris, France, IEEE, 2019, doi: 10.1109/CoDIT.2019.8820362

[23] Cheng, H.-Yang, L.-Liu, Z.: Survey on 3D Hand Gesture Recognition. IEEE Transactions on Circuits and Systems for Video Technology, Vol. 26, 2016, No. 9, pp. 1659-1673, doi: 10.1109/TCSVT.2015.2469551.

[24] Kishore, P. V. V.-Kameswari, P.S.-Niharika, K.-Tanuja, M.-Bindu, M.-Kumar, A. D.-Kumar, E. K.-Kiran, M. T.: Spatial Joint Features for 3D Human Skeletal Action Recognition System Using Spatial Graph Kernels. International Journal of Engineering and Technology, Vol. 7, 2018, No. 1.1, pp. 489-493, doi: 10.14419/ijet.v7i1.1.10152.

[25] Lun, R.-Zhao, W.: A Survey of Applications and Human Motion Recognition with Microsoft Kinect. International Journal of Pattern Recognition and Artificial Intelligence, Vol. 29, 2015, No. 5, Art. No. 1555008, doi: 10.1142/S0218001415550083.

[26] Sun, Y.-LI, C.-LI, G.-JiAng, G.-JiAng, D.-Liu, H.-Zheng, Z.Shu, W.: Gesture Recognition Based on Kinect and sEMG Signal Fusion. Mobile Networks and Applications, Vol. 23, 2018, No. 4, pp. 797-805, doi: 10.1007/s11036018-1008-0

[27] Cicirelli, G.-Attolico, C.-Guaragnella, C.-D’Orazio, T.: A KinectBased Gesture Recognition Approach for a Natural Human Robot Interface. International Journal of Advanced Robotic Systems, Vol. 12, 2015, No. 3, pp. 1-22, doi: $10.5772 / 59974$

[28] Lu, W.-Tong, Z.-Chu, J.: Dynamic Hand Gesture Recognition with Leap Motion Controller. IEEE Signal Processing Letters, Vol. 23, 2016, No. 9, pp. 1188-1192, doi: 10.1109/LSP.2016.2590470

[29] McCartney, R.-Yuan, J.-Bischof, H.-P.: Gesture Recognition with the Leap Motion Controller. Accessed from https://scholarworks.rit.edu/other/ 857. 2015.

[30] Artal-Sevil, J.S.-Montañés， J. L.-Acón， A.-Domínguez， J. A.: Control of a Bionic Hand Using Real-Time Gesture Recognition Techniques Through 
1234 N. Chivarov, D. Chikurtev, S. Chivarov, M. Pleva, S. Ondas, J. Juhar, K. Yovchev

Leap Motion Controller. 2018 XIII Technologies Applied to Electronics Teaching Conference (TAEE), La Laguna, Spain, IEEE, 2018, pp. 1-7, doi: 10.1109/TAEE.2018.8476122.

[31] Chivarov, N.-Shivarov, N.: Remote Control User Interfaces for Service Mobile Robots for Elderly Care. IFAC-PapersOnLine, Vol. 49, 2016, No. 29, pp. 73-76, doi: 10.1016/j.ifacol.2016.11.104.

[32] Chikurtev, D.-Yovchev, K.-Chikurtev, E.: Design and Functionality of Web User Interface for Control of Service Mobile Robot Through the Internet. Problems of Engineering Cybernetics and Robotics, Vol. 67, 2016, pp. 51-60.

[33] FlexBE, 2019. Retrieved July $1^{\text {st }}$, 2019, from http://wiki.ros.org/flexbe

[34] Buyval, A.-Afanasyev, I.-Magid, E.: Comparative Analysis of ROS-Based Monocular SLAM Methods for Indoor Navigation. Proceedings of the Ninth International Conference on Machine Vision (ICMV 2016), Proceedings of the SPIE, Vol. 10341, 2017, Art. No. 103411K, doi: 10.1117/12.2268809

[35] LI, Y.-ShI, C.: Localization and Navigation for Indoor Mobile Robot Based on ROS. 2018 Chinese Automation Congress (CAC), Xi'an, China, IEEE, 2018, pp. 1135-1139, doi: 10.1109/CAC.2018.8623225.

[36] Chikurtev, D.-Rangelov, I.-Chivarov, N.-Shivarov, N.-Gigov, A.: Control of Service Robot via Voice Commands. Problems of Engineering Cybernetics and Robotics, Vol. 69, 2017, pp. 62-67.

[37] Carruth, D. W.-Hudson, C. R.-Bethel, C. L.-Pleva, M.-Ondas, S.Juhar, J.: Using HMD for Immersive Training of Voice-Based Operation of Small Unmanned Ground Vehicles. In: Chen, J., Fragomeni, G. (Eds.): Virtual, Augmented and Mixed Reality. Applications and Case Studies (HCII 2019). Springer, Cham, Lecture Notes in Computer Science, Vol. 11575, 2019, pp. 34-46, doi: 10.1007/9783-030-21565-1_3.

[38] Ondas, S.-Gurcik, M.: Domain-Specific Language Models Training Methodology for the In-Car Infotainment. Intelligent Decision Technologies, Vol. 11, 2017, No. 4, pp. 417-422, doi: 10.3233/IDT-170310.

[39] Machová, K.-Mikula, M.-Szabóová, M.-Mach, M.: Sentiment and Authority Analysis in Conversational Content. Computing and Informatics, Vol. 37, 2018, No. 3, pp. 737-758, doi: 10.4149/cai_2018_3_737

[40] Liao, Y.-F.-Pleva, M.-Hladek, D.-Stas, J.-Viszlay, P.-Lojka, M.JuhAR, J.: Gated Module Neural Network for Multilingual Speech Recognition. 2018 $11^{\text {th }}$ International Symposium on Chinese Spoken Language Processing (ISCSLP), Taipei City, Taiwan, IEEE, 2018, pp. 131-135, doi: 10.1109/ISCSLP.2018.8706679.

[41] Hudson, C.-Bethel, C. L.-Carruth, D. W.-Pleva, M.-Juhar, J.Ondas, S.: A Training Tool for Speech Driven Human-Robot Interaction Applications. $201715^{\text {th }}$ International Conference on Emerging eLearning Technologies and Applications (ICETA 2017), Danvers, IEEE, 2017, pp. 167-172, doi: 10.1109/ICETA.2017.8102488. 
[42] Ondáš, S.-Pleva, M.-Krištan, R.-HusovskÝ, R.-JuháR, J.: VoMiS The VoiceXML-Based Multimodal Interactive System for NAO Robot. 2018 IEEE World Symposium on Digital Intelligence for Systems and Machines (DISA 2018), IEEE, 2018, pp. 315-319, doi: 10.1109/DISA.2018.8490598.

[43] Chivarov, N.-Chivarov, S.-Yovchev, K.-Chikurtev, D.-Shivarov, N.: Intelligent Modular Service Mobile Robot ROBCO 12 for Elderly and Disabled Persons Care. $201423^{\text {rd }}$ International Conference on Robotics in AlpeAdria-Danube Region (RAAD), Smolenice, Slovakia, 2014, pp. 343-348, doi: 10.1109/RAAD.2014.7002238.
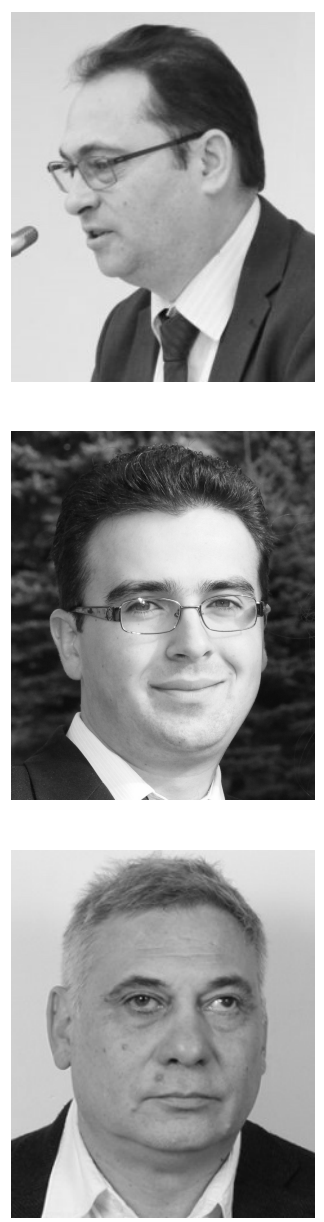

Nayden Chivarov received his Ph.D. degree from the Institute of Handling Device and Robotics, TU Vienna, Austria. Currently he is head of RILab i2030 in the Institute of Information and Communication Technologies - BAS and Associated Professor at the EPU. His research interests are: service robots, mechatronics, human-robot interaction, Industry 4.0, IoT.

Denis Chikurtev received his Ph.D. degree from the Institute of Information and Communication Technologies at Bulgarian Academy of Sciences in 2017. He is currently Conduct Researcher in the field of robotics and ICT and running teaching courses in internet technologies. His major research interests are in the area of robotics: hardware and software architectures, computer vision and indoor localization, and navigation applicable for industrial robotic manipulators or mobile service robots.

Stefan Chivarov is currently working towards his Ph.D. in the Institute of Mechanics and Mechatronics - IHRT, TU Vienna, Austria. His research interests are: software architecture, autonomous navigation and localization, robotics, smart technologies, IoT. 

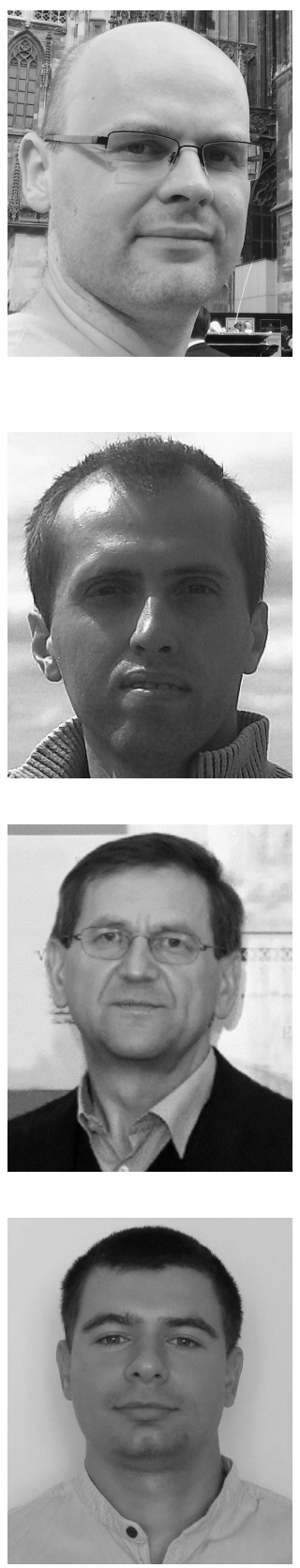

Matus Pleva received his Ph.D. degree in telecommunications at the Department of Electronics and Multimedia Communications of the Faculty of Electrical Engineering and Informatics at the Technical University of Košice in 2010. He works as Assistant Professor in the field of acoustic modeling, acoustic event detection, speaker recognition, speech processing, human-machine interaction, security and biometrics, networking, etc. He also participated in more than 30 national and international projects and COST actions. He has published over 100 technical papers in journals and conference proceedings.

Stanislav ONDAS graduated at the Department of Electronics and Multimedia Communications of the Faculty of Electrical Engineering and Informatics at the Technical University of Košice in 2004. He received his Ph.D. degree at the same department in the field of telecommunications in 2008. He is currently working as Assistant Professor in the Laboratory of Speech and Mobile Technologies at the same department. He is a specialist in the field of human-machine interaction, dialogue modelling and management, natural language processing and semantic analysis.

Jozef JuHAR graduated from the Technical University of Košice in 1980. He received his Ph.D. degree in radioelectronics from the Technical University of Košice in 1991, where he works as Full Professor at the Department of Electronics and Multimedia Communications. He is author and co-author of more than 200 scientific papers. His research interests include digital speech and audio processing, speech/speaker identification, speech synthesis, development in spoken dialogue and speech recognition systems in telecommunication networks.

Kaloyan Yovchev received his Ph.D. degree from the Faculty of Mathematics and Informatics at Sofia University "St. Kliment Ohridski" in 2018. He is currently teaching courses in computer science. His major research interest is development and implementation of iterative learning control methods for industrial robotic manipulators. Algorithms for robust control of unmanned robotic systems are also in his research area. 